\title{
Wide needle aspiration cytology in the diagnosis of lymphadenopathy in Zambia
}

\author{
P S Patil, C Bem
}

\begin{abstract}
Aims-To study the value of wide needle (19 gauge) aspiration cytology in the diagnosis of lymph node disease in Zambia in the absence of a trained cytologist.

Methods-Patients $(n=304)$ referred for surgical biopsy of an enlarged peripheral lymph node were studied prospectively. Surgical biopsy was routinely preceded by 19 gauge needle aspiration of the same node; aspirates were stained by haematoxylin and eosin and Ziehl Neelsen stains.

Results-Of 232 aspirates, 182 contained sufficient material for cytological characterisation. Tuberculosis was diagnosed or suspected in 122 of 126 aspirates with histologically confirmed tuberculous lymphadenitis; reactive follicular hyperplasia in 31 of 38 patients with primary HIV lymphadenopathy; malignancy in all five patients with malignant nodes; and Kaposi's disease in four of nine patients with this. Tuberculous lymphadenitis was falsely suspected in four patients, as was reactive follicular hyperplasia in four, and Kaposi's disease in four.

Conclusions-Wide needle aspiration cytology is useful in the diagnosis of lymphadenopathy in Central Africa, with the exception of lymphadenopathic Kaposi's disease.
\end{abstract}

(F Clin Pathol 1993;46:806-809)

Lymph node enlargement is becoming more and more common in Central Africa as a result of the spreading HIV pandemic. Increasing numbers of patients are presenting for surgical biopsy to exclude HIV associated tuberculous lymphadenitis and lymphadenopathic Kaposi's disease. ${ }^{1}$ Aspiration cytology offers a rapid and relatively cheap alternative to surgical biopsy in the diagnosis of lymph node disease, but remains underused in this region because of the scarcity of trained personnel, especially cytologists.

In the absence of a cytology service at our hospital we modified the fine needle aspiration cytology technique ${ }^{2-5}$ to suit the skills of a histopathologist. A wide bore (19 gauge) needle was used for aspiration to provide an increased yield of tissue and haematoxylin and eosin was used as the routine cytological stain. The technique is referred to as wide needle aspiration cytology and the following describes our observations on 232 lymph node aspirates using this technique.

\section{Methods}

In a prospective study covering the years 1989-1990, needle aspiration was carried out on patients undergoing diagnostic surgical biopsy of a peripheral lymph node in the University Teaching Hospital, Lusaka, Zambia. The patients were referred from all clinics and wards and were managed by one surgeon (CB). Cooperation with the chest clinic produced a bias towards patients presenting with suspected tuberculosis.

Aspiration was carried out using a 19 gauge needle and 20 millilitre syringe without the aid of a syringe holder ${ }^{5}$ by a surgeon (CB) with no previous experience of the technique. Aspiration was carried out under anaesthesia immediately before open surgical biopsy of the same node. The aspirate was spread on to two slides, air dried, and coded. One aspirate was subsequently stained with haematoxylin and eosin and the other with the Ziehl Neelsen stain. The staining techniques were identical with those used on histological sections.

In a consecutive series of patients, fine needle (21 gauge) aspiration was carried out before wide needle aspiration. The same histopathologist (PSP) examined both aspirates and histopathology sections, but to avoid bias the aspirates were examined independently of the histological diagnoses and were not used for clinical management. On completion of the study, aspirates were reexamined and compared with histological results.

Criteria for cytodiagnosis were the same as those used on histological sections. Histological diagnosis was made on the basis of examination of the stained sections, with additional sections stained by periodic acid schiff and Grocott's methods, when indicated. Part of each surgically removed lymph node was routinely cultured for Mycobacterium tuberculosis. HIV-1 serology was tested on all consenting patients by competitive recombinant enzyme linked immunosorbent assay (ELISA) (Wellcozyme, Wellcome Diagnostics, Dartford, Kent).

Statistical analyses were carried out with the help of Epi info, version 5 (USD Inc., Stone Mountain, Georgia, USA) and the CIA Microcomputer Program (British Medical Fournal, Martin Gardner, 1991).

Ethical approval for the study was given by 
Table 1 Histology, HIV-1 serology, and quality of aspiration by wide needle

\begin{tabular}{lcll}
\hline Histology & $n=$ & $\begin{array}{l}\text { HIV-1t } \\
\text { serology }\end{array}$ & $\begin{array}{l}\text { Good quality } \\
\text { aspirates }\end{array}$ \\
\hline Tuberculous lymphadenitis & 155 & $130 / 152$ & $\begin{array}{l}126 / 155 \\
(81 \cdot 3 \%)\end{array}$ \\
Primary HIV lymphadenopathyt & 47 & $\begin{array}{l}47 / 47 \\
(100 \%)\end{array}$ & $\begin{array}{l}38 / 47 \\
(80 \cdot 1 \%)\end{array}$ \\
Kaposi's disease & 18 & $18 / 18$ & $9 / 18$ \\
Lymphoma & 5 & $(100 \%)$ & $(50 \cdot 0 \%)$ \\
Malignant metastasis & 4 & $(40 \cdot 0 \%)$ & $3 / 5$ \\
Other pathology & 4 & $1 / 3$ & $(60 \cdot 0 \%)$ \\
Total & $43 \cdot 3 \%)$ & $2 / 4$ \\
& $232^{\star \star}$ & $3 / 4$ & $(50 \cdot 0 \%)$ \\
& & $200 / 228$ & $(100 \%)$ \\
\hline
\end{tabular}

Notes:

^Acid fast counts per high power field on histology of tuberculous lymphadenitis: 76 nodes $0 / \mathrm{hpf} ; 34$ nodes, $<1 / \mathrm{hpf} ; 12$ nodes, $1-10 / \mathrm{hpf} ; 9$ nodes, $11-100 / \mathrm{hpf} ; 10$ nodes, $>100 / \mathrm{hpf} ; 14$ nodes, not available.

tPrimary HIV lymphadenopathy was diagnosed on the basis of characteristic histology ${ }^{67}$ in association with positive HIV serology.

‡Other pathology: non-HIV related follicular hyperplasia $n=1$; extraparotid lymphoepithelial cysts $n=2^{8}$; Castleman's disease $n=1$.

cysts $n=2^{8}$; Castleman's disease $n=1$.
$\star \star$ One patient with double lymph node pathology: tuberculous lymphadenitis in association with Kaposi's disease (HIV seropositive, poor quality aspirate)

the Ethics Subcommittee of the National AIDS Surveillance Committee of the Ministry of Health of the Republic of Zambia.

\section{Results}

Material was aspirated from 270 of 304 patients (all indigenous Zambians) undergoing peripheral lymph node biopsy. Aspiration was unsuccessful in the remaining 34 patients, because the nodes were either too small or too deep.

Both haematoxylin and eosin and Ziehl Neelsen stained aspirates were available at final review for a total of 232 patients (mean age 30 years, range 2-67 years, 115 males, 117 females) who form the material for the subsequent analysis. The sites of aspiration were: neck $n=150$; axilla $n=43$; groin $\mathrm{n}=33$; epitrochlear $\mathrm{n}=6$. The histological findings are shown in table 1 . Culture for $M$ tuberculosis was positive in 85 of 148 $(57.4 \%, 95 \%$ confidence intervals 49.5 $65 \cdot 4 \%$ ) nodes with histologically diagnosed tuberculous lymphadenitis subjected to culture for mycobacteria. No atypical mycobacteria were grown. Two hundred of 228 $(87 \cdot 7 \%, 95 \%$ CI $83 \cdot 4-92 \%)$ patients tested were HIV-1 seropositive (table 1).

Overall, $182 / 232 \quad(78.4 \%, \quad 95 \% \quad$ CI $73 \cdot 2 \%-83 \cdot 7 \%$ ) aspirates showed sufficient

Table 2 Cytological findings in good quality wide needle aspirates

\begin{tabular}{|c|c|c|}
\hline Cytological findings & $n=$ & Histological diagnosis \\
\hline $\begin{array}{l}1 \text { Acid fast bacilli positive } \\
2 \text { Acid fast bacilli negative, caseation positive } \\
3 \text { Acid fast bacilli negative, caseation negative: }\end{array}$ & $\begin{array}{l}43 \\
75\end{array}$ & 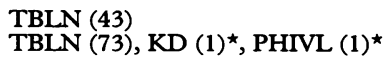 \\
\hline $\begin{array}{l}\text { (a) Granuloma(s) or giant cell(s) } \\
\text { (b) Reactive lymphoid hyperplasia } \\
\text { (c) Fascicles of spindle cells }\end{array}$ & $\begin{array}{l}8 \\
37 \\
8\end{array}$ & $\begin{array}{l}\text { TBLN (6), KD (1) }{ }^{\star}, \text { PHIVL (1) } \\
\text { PHIVL (31), TBLN (3), other (3) } \\
\text { KD (4), TBLN (2), PHIVL (1) } \\
\text { other (1) }\end{array}$ \\
\hline (d) Suspicious, malignant cells & 7 & Malignancy (5), KD (1), TBLN (1) \\
\hline
\end{tabular}

AFB, acid fast bacilli; TBLN, tuberculous lymphadenitis; PHIVL, primary HIV lymphadenopathy; KD, Kaposi's disease.

${ }^{\star}$ False positive results (see text). material for cytological characterisation, and these are referred to as good quality aspirates (table 1). Not all aspirates judged to be good quality provided diagnostic material. The quality of the aspirates improved during the study, 65 of the first 100 aspirates and 88 of the last 100 aspirates being of good quality $\left(\chi^{2}=14 \cdot 71,1 \mathrm{df}, \mathrm{p}=0 \cdot 0001\right)$. Poor quality aspirates showed scanty material and were often heavily bloodstained. Aspiration from a node with Kaposi's disease was especially likely to give a poor quality aspirate unfit for diagnosis (nine of 18 from nodes with Kaposi's disease, 41 of 214 from nodes without Kaposi's disease; Fisher's exact test, $\mathrm{p}=0.005$ ).

The findings from cytological examination of the good quality aspirates are summarised in table 2.

Tuberculosis can be diagnosed by the presence of acid fast bacilli on cytology and suspected by the presence of caseation, granulomas, or giant cells. Acid fast bacilli were present in 43 of $126(34.1 \%, 95 \% \mathrm{CI}$ 25.8-42.4\%) good quality Ziehl Neelsen stained aspirates from tuberculous nodes with the following counts per high power field $(\times 400)$ : 16 aspirates $<1 / \mathrm{hpf} ; 11$ aspirates 1-10/hpf; seven aspirates $11-100 / \mathrm{hpf} ; 9$ aspirates, $>100 / \mathrm{hpf}$.

Caseation was present in $114(90.5 \%, 95 \%$ CI 85.4-95.0\%) granulomas in $27(21.4 \%$, 95\% CI $14 \cdot 3-28 \cdot 6 \%$ ) and giant cells in 14 $(11 \cdot 1 \%$, CI $5 \cdot 6-16.6 \%)$ of the 126 good quality haematoxylin stained aspirates from tuberculous nodes. Caseation was present in all but two acid fast bacilli positive good quality aspirates from tuberculous nodes and in 73 of $83(88 \%, 95 \%$ CI $79-94 \cdot 1 \%)$ acid fast bacilli negative good quality aspirates from tuberculous nodes. Granulomas or giant cells, or both, were present in six of the remaining ten acid fast bacilli negative, caseation negative good quality aspirates from tuberculous nodes. In only four of $126(3.2 \%, 95 \%$ CI $0 \cdot 9-7.9 \%$ ) good quality aspirates from tuberculous lymph nodes did the diagnosis remain unsuspected on cytology.

Review of histological sections and aspiration slides showed that there were four false positive diagnoses of tuberculosis on cytology (table 2). In two aspirates amorphous material enmeshed in small fibrin clumps was mistaken as a focus of caseation; in one aspirate a cluster of histiocytes was called a granuloma, and in one aspirate an artefact was described as a giant cell. No Warthin-Finkeldey-like giant cells, described in primary HIV lymphadenopathy, ${ }^{67}$ were seen.

A cytological diagnosis of reactive lymphoid hyperplasia was made in 37 aspirates. This represented 31 of $38(81.6 \%, 95 \%$ CI $65 \cdot 7-92 \cdot 3 \%$ ) good quality aspirates from patients with primary HIV lymphadenopathy, one aspirate from a node with a lymphoepithelial cyst, a condition recently described in HIV disease ${ }^{8}$ and one aspirate from the one node with non-HIV related follicular hyperplasia. Reactive lymphoid hyperplasia was wrongly diagnosed in three HIV positive 
tuberculous lymph nodes (with no aspirate evidence of tuberculosis) and in one patient with Kaposi's disease. No cytological evidence of toxoplasmosis was detected in the aspirates.

Fascicles of spindle cells were seen in eight aspirates. Only four of the nine good quality aspirates from Kaposi's disease showed spindle cells. Morphologically, these could not be distinguished from clusters of cells presumed to be fibrocellular material, aspirated from four nodes with no histological evidence of Kaposi's disease (two nodes with tuberculous lymhadenitis, one node with primary HIV lymphadenopathy, and one node with nonHIV related follicular hyperplasia).

Malignant cells were identified in all five good quality aspirates from malignant nodes (two nodes with metastatic disease, three nodes with non-Hodgkin's lymphoma). Plump hyperchromatic spindle cells, reported as possibly malignant, were reported from one aspirate from a node with Kaposi's disease and one node with tuberculosis.

A distinctly bloody aspirate was noted in a total of 62 with or without material adequate for cytological characterisation. Such an aspirate was more likely to have come from a node with Kaposi's disease: 14 of the 18 aspirates from nodes with Kaposi's disease and 48 of the 214 aspirates from non-Kaposi's disease nodes were distinctly bloodstained on cytological examination $\left(\chi^{2}=25.97, p<0.0001\right)$.

Thirty eight patients underwent aspiration both by 21 gauge and 19 gauge needle. The proportion of good quality aspirates by 21 gauge aspiration was 18 of $38(47 \cdot 4 \%)$ and by 19 gauge needle 29 of $38(76 \cdot 3 \%)$, a difference of $28 \cdot 9 \%$ (95\% CI $7 \cdot 5-38 \cdot 2 \%$ ).

\section{Discussion}

The efficacy of aspiration cytology in the management of disease rests on four skillsnamely, aspiration, staining, cytological interpretation and clinical application.

Diagnostic needle aspiration was introduced during the $1920 \mathrm{~s}-30$ s using a wide bore needle of 18 gauge size $^{23}$ and has evolved into the modern technique known as fine needle aspiration cytology (FNAC),

Table 3 Predictive value of wide needle aspiration cytology in diagnosis of lymph node disease in Lusaka

\begin{tabular}{|c|c|c|c|c|}
\hline Pathology & $T B L N$ & PHIVL & $\begin{array}{l}\text { Kaposi's } \\
\text { disease }\end{array}$ & Malignancy \\
\hline $\begin{array}{l}\text { Cytological feature } \\
\text { True positive } \\
\text { False positive }\end{array}$ & $\begin{array}{l}\mathrm{AFB} / \mathrm{Cas} / \mathrm{Gr} / \mathrm{GC}^{\star} \\
122 \\
4\end{array}$ & $\begin{array}{l}\text { RLH } \\
31 \\
6\end{array}$ & $\begin{array}{c}\text { SC } \\
\mathbf{4} \\
\mathbf{4}\end{array}$ & $\begin{array}{c}\text { MC } \\
5 \\
2 \dagger\end{array}$ \\
\hline $\begin{array}{l}\text { Good quality slides } \\
\text { Sensitivity } \\
\text { Specificity } \\
\text { PPV } \\
\text { All slides }\end{array}$ & $\begin{array}{l}96 \cdot 8 \% \\
92 \cdot 9 \% \\
96 \cdot 8 \%\end{array}$ & $\begin{array}{l}81 \cdot 6 \% \\
95 \cdot 8 \% \\
83 \cdot 8 \%\end{array}$ & $\begin{array}{l}44 \cdot 4 \% \\
97 \cdot 7 \% \\
50 \cdot 0 \%\end{array}$ & $\begin{array}{c}100 \% \\
98.9 \% \\
71 \cdot 4 \%\end{array}$ \\
\hline $\begin{array}{l}\text { Sensitivity } \\
\text { Specificity } \\
\text { PPV }\end{array}$ & $\begin{array}{l}\mathbf{7 8 \cdot 7} \% \\
94 \cdot 8 \% \\
96 \cdot 8 \%\end{array}$ & $\begin{array}{l}66 \cdot 0 \% \\
96 \cdot 8 \% \\
83 \cdot 8 \%\end{array}$ & $\begin{array}{l}28 \cdot 6 \% \\
98 \cdot 1 \% \\
50 \cdot 0 \%\end{array}$ & $\begin{array}{l}55 \cdot 6 \% \\
99 \cdot 1 \% \\
71 \cdot 4 \%\end{array}$ \\
\hline
\end{tabular}

TBLN, tuberculous lymphadenitis; PHIVL, primary HIV lymphadenopathy; AFB, acid fas bacilli; Cas, caseation; Gr, granuloma; GC, giant cell; $R L H$, reactive lymphoid hyperplasia; SC spindle cells; MC, cells suspicious of malignancy; PPV, positive predictive value.

$\star$ One or more of these features present.

tKaposi's disease is not considered a malignancy ${ }^{1}$. using needles of $21-25$ gauge. ${ }^{2-7}$ This is an exacting skill, requiring specialist cytological training, ${ }^{2}$ and we wondered if aspiration diagnosis could be facilitated by providing more material for examination. We therefore returned to the use of a wider bore needle.

Overall, sufficient material for cytological characterisation was present in $78.4 \%$ of aspirates and this improved during the study. Comparison of the diagnostic yield of FNAC and wide needle aspiration cytology (WNAC) is difficult because in reported series of lymph node FNAC, ${ }^{39-12}$ the pathology was different, aspirates were examined by experienced cytologists, and exclusion of malignant disease was often the most important object of the investigation. The small numbers of patients in our study who underwent both 21 gauge and 19 gauge aspiration does suggest, however, that WNAC is associated with an increased diagnostic yield.

The use of haematoxylin and eosin for cytological staining has the advantage that the aspirate smear is familiar to the histopathologist practising cytology, and in laboratories where resources are scarce, there is the advantage that no additional reagents are needed. Haematoxylin and eosin is cheap, easily prepared, and allows aspirate slides to be stained and ready for examination in 15 minutes.

Though used by two investigators inexperienced in its use, lymph node WNAC in Zambia provided adequate material for effective interpretation in most patients and would have spared those patients the need for surgery and saved resources. This is particularly true when only good quality aspirates are considered (table 3 ).

Tuberculous lymphadenitis could have been diagnosed or suspected on cytology by the presence of acid fast bacilli, caseation, granulomas or giant cells in 122 of 126 $(96.8 \%)$ of histologically proved tuberculous lymph nodes with good quality aspirates. The small number of false positive results occurred where undue importance was given to an isolated giant cell and to a cluster of histiocytes interpreted as a granuloma. On two occasions small foci of amorphous material enmeshed in fibrin were interpreted as caseation: with greater experience they would not have been taken as such.

The most reliable cytological feature for the diagnosis of tuberculous lymphadenitis is the presence of acid fast bacilli, but the most common cytological feature associated with it in this study was caseation. Caseation was present in $73.6 \%$ of all aspirates $(90.5 \%$ of good quality aspirates) from tuberculous lymph nodes, which compares with $25-40 \%$ of fine needle aspirates from tuberculous lymph nodes in India and Hong Kong. ${ }^{13-15}$ One reason for the increased yield of caseation in our study may have been that the wider bore needle permits easier aspiration of thick, viscous, caseating material.

Malignancy was diagnosed or suspected in all malignant lymph nodes where the aspirate was of adequate quality. 
Primary HIV lymphadenopathy was suggested by an aspirate showing reactive lymphoid hyperplasia in a patient population among whom HIV infection was the most common cause of reactive follicular hyperplasia. Similar appearances were seen in patients with secondary lymph node disorders associated with HIV infection and represent failure of the needle to sample that part of the node containing the secondary disease. Therefore, a presumptive cytological diagnosis of primary HIV lymphadenopathy must be supported by clinical findings and positive HIV serology. The primary importance of WNAC in HIV positive patients remains, however, the exclusion of treatable disease, the most important of which is tuberculosis.

The diagnosis of lymphadenopathic Kaposi's disease in our study was unsatisfactory. A good quality aspirate was particularly unlikely from a lymph node with Kaposi's disease. This is perhaps because the spindle cells are too cohesive to be sucked into the needle, and wide needle aspiration from this vasoactive disorder is especially likely to be bloody. Clusters of fibrocellular material from occasional non-Kaposi's nodes were also indistinguishable from the fascicles observed in aspirates from nodal Kaposi's disease. This is perhaps because the wide needle sometimes permits aspiration of the connective tissue of the lymph node; the presence of spindle cells on FNAC his reliably been found to predict the presence of Kaposi's disease in the United States. ${ }^{16}$ The possibility that the nodes in our study contained isolated foci of Kaposi's disease cannot be excluded as the surgically biopsied nodes were not subjected to section at multiple levels.

In conclusion, we suggest that WNAC is a less rigorous technique than FNAC for both the aspirator and cytologist. We think it has a particularly valuable role in regions where diagnostic facilities are limited, as it allows a reliable cytology service to be provided by a histopathologist, with the exception of the diagnosis of nodal Kaposi's disease. With increasing exposure to cytological material, transition to FNAC could be made later as WNAC is associated with some disadvan- tages. Patient comfort usually requires infiltration of the skin for WNAC under local anaesthetic and, though very rare, the isolated cases of needle track malignant seeding have been reported from the use of wide needle aspiration. ${ }^{4}$

We are grateful for help and cooperation from: Dr A M Elliott of the University Teaching Hospital, Lusaka, and the London School of Hygiene and Tropical Medicine, Dr N Luo of the Department of Immunology of the University Teaching Hospital, Mr K M Namambo of the Chest Diseases Laboratory, Lusaka, and Dr H Bharucha of the Department of Pathology of the Queen's University, Belfast. We are grateful for financial ful for financial assistance from the Commission of the European Communities, contract TS2.004.UK(H) and for Department of Dermatovenereology, University Teaching Hospital.

1 Bayley AC, Lucas SB. Kaposi's sarcoma or Kaposi's disease? A Personal reappraisal. In: Fletcher CDM, McKee PH, Eds. Pathobiology of soft tissue tumours. Edinburgh: Churchill Livingstone, 1990:141-63.

2 Orell SR. Fine needle aspiration biopsy in perspective. Pathology 1982;14:113-4.

3 Bottles K, Miller TR, Cohen MB, Ljung B. Fine needle aspiration biopsy: has its time come? Am F Med 1986; 81:525-31.

4 Philips JN, Goodman BN. Clinching the diagnosis: fine needle aspiration cytology. Pathology 1987;19:371-6.

5 Melcher D, Linehan J, Smith R. Practical Aspiration Cytology. Edinburgh, Churchill Livingstone, 1984.

6 Ewing EP, Chandler FW, Spira TJ, Brynes RK, Chan WC. Primary lymph node pathology in AIDS and AIDS-related lymphadenopathy. Arch Pathol Lab Med 1985;109:977-81.

7 Racz P, Tenner-Racz K, Kahl C, Feller AC, Kern P, Dietrich M. Spectrum of morphologic changes in lymph nodes from patients with AIDS or AIDS-related comnodes from patients with AIDS or AII
plexes. Progr Allergy 1986;37:81-181.

plexes. Progr Allergy 1986;37:81-181.
8 Bem C, Bharucha H, Patil PS. Parotid disease and HIV infection in Zambia. Br ₹ Surg 1992;79:768-70.

9 Frable WJ. Thin-needle aspiration biopsy: a personal experience with 469 cases. Am $\mathcal{f}$ Clin Pathol 1976;65: 168-81.

10 Kline TS, Kannan V, Kline IK. Lymphadenopathy and aspiration biopsy cytology: review of 376 superficial nodes. Cancer 1984;54:1076-81.

11 Patra AK, Nanda BK, Mohapatra BK, Panda AK. Diagnosis of lymphadenopathy by fine needle aspiration cytology. Indian $\mathcal{7}$ Pathol Microbiol 1983;26:273-8.

12 Singh A, Singh M, Gupta SK. Role of fine needle aspiration cytology in the diagnosis of lymphadenopathy. tion cytology in the diagn

13 Metre MS, Jayaram G. Acid-fast bacilli in aspiration smears from tuberculous lymph nodes: an analysis of smears from tuberculous lymph nod
255 cases. Acta Cytol 1987;31:17-9.

14 Dandapat MC, Panda BK, Patra AK, Acharya N. Diagnosis of tubercular lymphadenitis by fine needle aspiration cytology. Ind $f$ Tuberc 1987;34:139-42.

15 Lau SK, Wei WI, Hsu C, Engzell UCG. Fine needle aspiration biopsy of tuberculous cervical lymphadenopathy. Aust NZ $\mathcal{Y}$ Surg 1988;58:947-50.

16 Hales $M$, Bottles $K$, Miller $T$, Donegan E, Ljung B. Diagnosis of Kaposi's sarcoma by fine-needle aspiration biopsy. Am f Clin Pathol 1987;88:20-5. 